Printed in Great Britain

\title{
NEUROMUSCULAR ACTIVITY IN SEA ANEMONE CALLIACTIS PARASITICA (COUCH)
}

\author{
By MARY NeEDLER ${ }^{\star}$ and D. M. Ross \\ Department of Zoology and Comparative Anatomy, University College, London
}

(With Text-figs. I-7)

In a recent study (Ross, 1957) isolated marginal sphincter preparations of the sea anemone, Calliactis parasitica, showed two distinct responses to stimulation: (I) a quick contraction in response to stimuli at frequencies from 0.2 to $3.0 \mathrm{sec}$, the facilitated response of Pantin (1935 a) beginning only on the second stimulus of a series, with a latent period of about O.I sec; (2) a smooth slow contraction in response to stimuli at lower frequencies, with a latent period of not less than $30 \mathrm{sec}$, and beginning only after several stimuli. This slow movement of the marginal region had not been detected in work on whole animals, but it is similar to the contractions of the 'slow' muscles of Calliactis (Pantin, 1935 b) and Metridium (Batham \& Pantin, 1950 a). However, Calliactis sphincter shows almost no spontaneous activity.

Both quick and slow contractions had previously been observed in excised sphincters and mesenteric retractors of Metridium (Batham \& Pantin, 1954), the chief muscles utilized in the closure of that animal. In all these cases, the quick contraction would seem to be a specialized mechanism for sudden withdrawal superimposed upon, and perhaps developed from, a more primitive and general slow contractile mechanism. As part of a programme to investigate and compare the properties of quick and slow contractions in these muscles it seemed desirable to see what part, if any, slow contractions of the marginal sphincter play in the life of Calliactis. This involved us in a wider study of the neuromuscular activity of this anemone which normally lives on shells inhabited by the hermit crab, Eupagurus bernhardus. We report the results here as an additional contribution to the analysis of behaviour in the Actinozoa, supplementing the similar studies on Metridium by Batham \& Pantin $(1950 a, b)$.

\section{METHODS}

As in the work of Batham \& Pantin (1950b, 1954), this investigation required the use of several methods of recording and observing the movements of the animals. Kymograph records, together with visual and photographic records, were used for information on the frequency, size and form of certain periodic activities. A short film record supplemented these observations. Preparations

\footnotetext{
* Present Address: Department of Zoology, University of California, Los Angeles, U.S.A.
} 
of Calliactis consisting of several rings joined to a strip of column were studied for information on innate rhythmic activity and on the timing and co-ordination of movements at different levels of the column. Responses to electrical and other forms of stimulation were also studied, both in whole animals and preparations, in order to see how they resembled the natural movements without stimulation.

\section{RESULTS}

With its thicker and tougher integument and mesogloea, Calliactis does not show the extremes of shape and posture observed in Metridium. It can close down to a flattish cone but it never assumes the wrinkled and shrivelled appearance that is seen so commonly in Metridium. It can extend itself or bend to the side only to moderate degrees, and local contractions of the circular muscle of the column never produce in Calliactis the conspicuous deep furrows so clearly seen in Metridium (Batham \& Pantin, I950a). Moreover, unlike Metridium, Calliactis does not 'walk'; once it becomes firmly fixed on a plate or a stone, it may remain in the same place for months. It only moves by detaching itself altogether and creeping or rolling about the tank in a prone position.

Close observation of Calliactis shows, as Batham \& Pantin (1950b) have remarked, that this anemone, like Metridium, is in a state of continual activity. Although most of the movement is too slow to be visible and the changes in appearance are less conspicuous than in Metridium, shape and posture are often quite different from one minute to the next. Moreover, there are periods when an exceptional amount of activity seems to be taking place and these periods are repeated at regular intervals.

Besides slowly changing in posture and shape, Calliactis closes up from time to time, even in the absence of any obvious stimulus. In order to find out how often it does so, ten animals were observed closely for 5 min periods at hourly intervals, ten times every day for a week. This gave us a record of the number of closing movements in $350 \mathrm{~min}$ observation time. In fourteen of the seventy observation periods, all ten animals remained open the whole time. The total number of closures recorded was I08, and on two occasions, four different animals exhibited closing movements during the whole or part of the same $5 \mathrm{~min}$ period. There were big differences in individuals. One was closed twenty-one times; another was never seen to close during the seventy observation periods. Thus closure may occur in some animals several times an hour, but in the majority it occurs with a frequency of about once an hour, and in some it is a very rare event indeed.

Two kinds of closing movement were evident in these animals. Some, like the responses to mechanical and electrical stimulation, were of short duration, with little loss of water or change of volume and the animal expanded again 
within a minute or two (Pantin, 1940; Chapman, 1949). Others were longlasting. Animals closed at the beginning were frequently still closed at the end of an observation period, and some of these were also closed at the next and even later observation periods as well. From occasional checks in between, we know that in many of these cases no opening occurred in the intervening periods. These closures, which may be sustained for several hours, comprised about one-third of the total, and since the contraction of the animal in these closures was more complete and water loss greater, relaxation and opening were very much slower than in the familiar closing responses of short duration.

Since one of the objects of the investigation was to find out if slow contractions of the sphincter play any part in the normal behaviour of Calliactis, the closing movements were followed closely to see if slow contractions of the margin could be detected in them. It may be significant in this connexion that the first stages of these closing movements were not often seen. Closures beginning with a quick contraction were spotted only seven times. In the remaining 67 closures, the animals were already partly closed before any movement was detected. This may mean either that an observer tends to miss an initial quick contraction or that the movement begins slowly and only becomes apparent later. Subsequently, smooth flowing movements of the margin could be seen taking part in completing the closing movement, though occasionally the jerky movements of a quick contraction might be inserted into the sequence. Relaxation after many of these closures and partial closures was slow, resembling the time-course of relaxation after slow contractions of sphincter preparations (Ross, 1957) rather than the dramatic opening which follows quick closures. We concluded, therefore, that slow contractions of the sphincter contribute substantially to the spontaneous closing movements that occur from time to time in unstimulated Calliactis.

Closing movements are not the only feature of the behaviour of unstimulated Calliactis. The margin is frequently thrown into graceful folds by local contractions in certain radii. Changes in length and local constriction and peristalsis in the column produce a variety of shapes, some of which are illustrated in Fig. 6. Defaecation, with its gaping mouth and shapeless appearance, is easily recognized in Calliactis as in Metridium (Batham \& Pantin, 1950). Many of these phases involve considerable shortening of the margin without quick sphincter contractions. Thus slow movements of the sphincter seem to be involved in these activities also.

\section{RHYTHMIC CYCLES OF ACTIVITY IN CALLIACTIS}

Some of the movements seen in Calliactis over a period seemed to have a repetitive character. Kymograph recordings gave clear evidence of this, and often showed a slow rhythm of remarkable regularity. Fig. I is a $3 \mathrm{hr}$ record 
of Calliactis attached to a light gimbal lever. The big downward movements represent slow contractions of the submarginal and marginal regions culminating in a shortening of the column. This took 3-4 min to complete and was repeated every IO-I $5 \mathrm{~min}$. The regularity, frequency and size of the main movement in the cycle vary greatly from one animal to another, and in the same animal at different times. But repetitive movements are always a feature of the records and they usually have marginal and submarginal components which are repeated several times per hour.

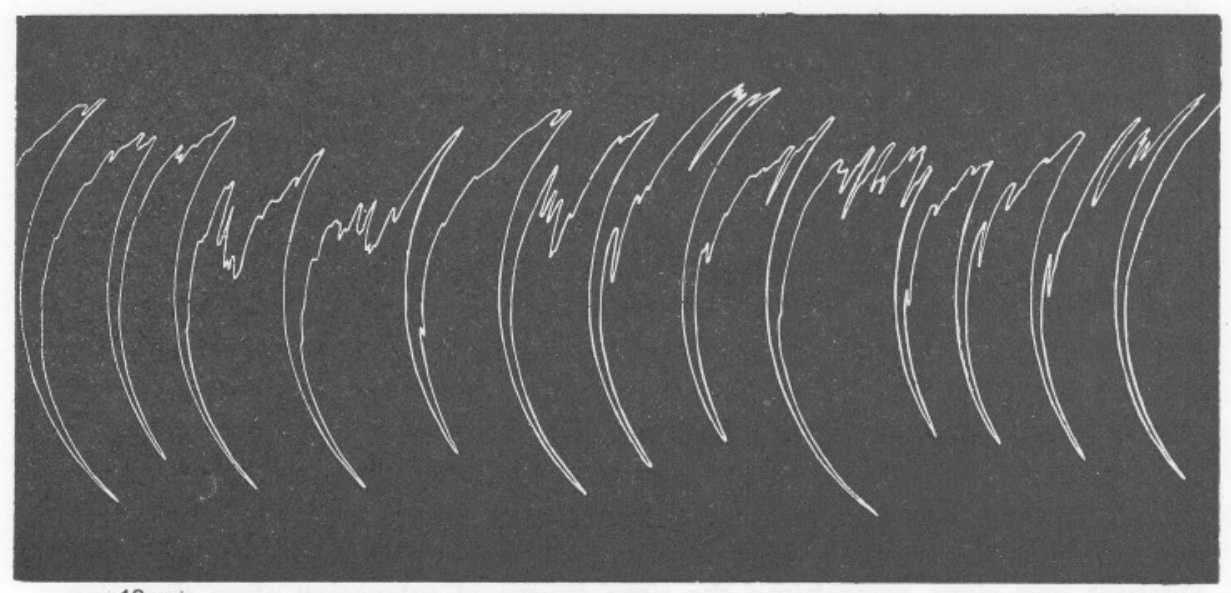

$10 \mathrm{~min}$

Fig. I. Continuous record of movements of Calliactis during $3 \mathrm{~h}$. Thread inserted in margin and attached obliquely to gimbal lever.

It is not easy to relate these cycles of activity to the closing movements which occur intermittently in unstimulated animals. The cycles recorded on the kymograph occur more frequently than visible closing movements. Moreover, the recorded movements sometimes produce a condition corresponding to partial closure, but full closure and sustained closure are almost never seen, though this may simply be due to the pull of the lever. We know also that a good many animals show visible closing movements very infrequently, yet in our experience all Calliactis give evidence of repeated activity cycles on the kymograph. It seems to us that the closing movements may be regarded as one form that the main movement of the repetitive cycle may take in some animals at certain times. Further data on this matter will be presented later.

The detailed picture of these low-frequency 'beats' varies greatly from one animal to another and from day to day in the same animal. There are periods, however, usually lasting several hours, when the rate is constant and then the form is remarkably constant too. Each cycle normally consists of one 
main series of contractions followed by a partial relaxation and then by one or more subsidiary cycles. In typical cycles, like those shown in Figs. I-3, ten or more distinct movements may be involved. The main movement often begins, as in Fig. 2, with a small quick contraction of the sphincter, and even when this does not appear in the record, a slight twitch or bristling of the tentacles and marginal edge is often detected visually, just before the slow downward movement of the lever starts. The main components in this movement are a slow contraction of the submarginal region, and an even shortening of the column and depression of the disk, presumably by the longitudinal mesenteric and parietal muscles. In Fig. 3 the final pull on the lever is due to a slow sphincter contraction which is powerful enough to mask the relaxation of the column taking place at the same time. Most of the big movements of the cycles consist of combinations of at least two of these components but a variety of additional minor movements may be involved in various sequences.

In the subsequent relaxation, the column lengthens, presumably by the contraction of the circular muscles, but before relaxation is complete it is generally interrupted by a subsidiary cycle. This is characterized by the constriction and twisting of the column at the level of the cinclides, initiating a peristaltic wave of contraction which moves up the column and which may involve the margin.

Electrical stimuli giving a quick contraction of the sphincter can reproduce a good deal of this activity, and generally initiate a complete cycle conforming to the pattern of repetitive activity at the time. This suggests that the small sphincter contraction or tentacular twitching, so often seen just prior to the beginning of a cycle, may be a signal that the cycle is about to start, or may act as its trigger. Low-frequency stimuli, which elicit only slow contractions, do not initiate new cycles, possibly because several muscle systems which normally work independently are brought into action simultaneously so that the sequence is disrupted.

\section{Activities of isolated preparations of Calliactis}

Two kinds of preparation have been studied: (I) simple rings taken from the column at all levels; (2) preparations consisting of three loops taken from the margin, the submarginal region and the lower column at the level of the cinclides, joined together by a connecting strip of column.

The simple ring preparations showed that the circular muscle of the column is rhythmically active at all levels below the sphincter. Moreover, the timing of the 'beats' is very close to that observed in the movements of whole animals, usually with several main movements and rather more subsidiary movements occurring per hour (Fig. 4). Such rings give slow contractions to stimuli like those obtained from Metridium preparations (Batham \& Pantin, I954), and these contractions are similar to the main movements of the cycles 

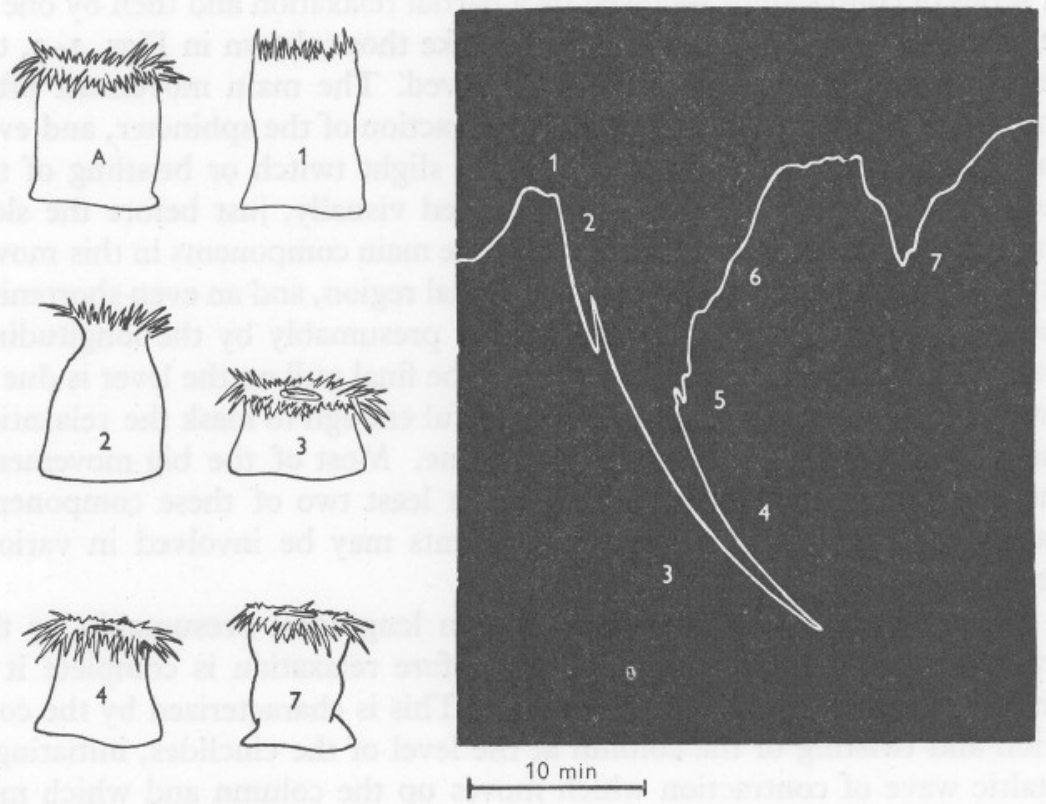

Fig. 2. Record of a single cycle in the rhythmic activity of Calliactis during $35 \mathrm{~min}$. Numbers refer to the following distinct stages recognized visually and depicted in the accompanying sketches: (I) quick sphincter contraction; (2) submarginal contraction; (3) column shortening; (4) column extension; (5) brief submarginal contraction followed by (6) expansion of disc; (7) subsidiary cycle with transitory changes in length and peristaltic movements of the column. A shows the animal in the non-active stage.
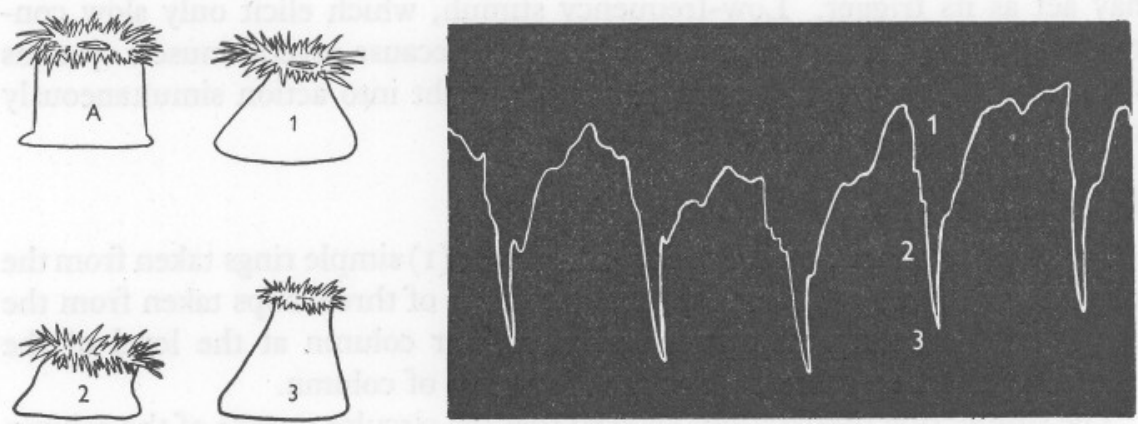

$$
10 \mathrm{~min}
$$

Fig. 3. Record of five cycles in the rhythmic activity of Calliactis during $39 \mathrm{~min}$. Numbers on record and sketches refer to the following distinct stages in the movement: (I) submarginal contraction; (2) column shortening; (3) slow sphincter contraction merging into column extension. A shows the non-active stage. 
(Fig. 4). As a rule ten or more stimuli are required to start such a movement and the latent periods are usually at least I min.

The triple-loop preparations gave evidence of co-ordinated activities. Movements occurred about every I0-20 $\mathrm{min}$ as in the intact animal, and in these movements, all three loops came into action in a definite sequence. Since the longitudinal strip of column joining the loops was firmly fixed to a cork plate with pins and held firmly in position by strips of glass pressed down by rubber bands, there was no possibility of the movement of one loop pulling mechanically on the next. The co-ordination seems to be based,

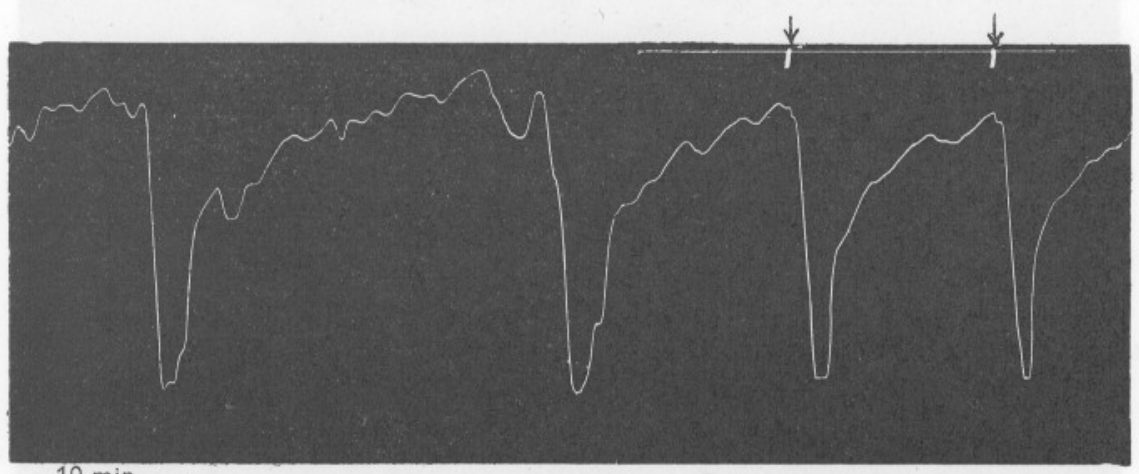

$10 \mathrm{~min}$

A

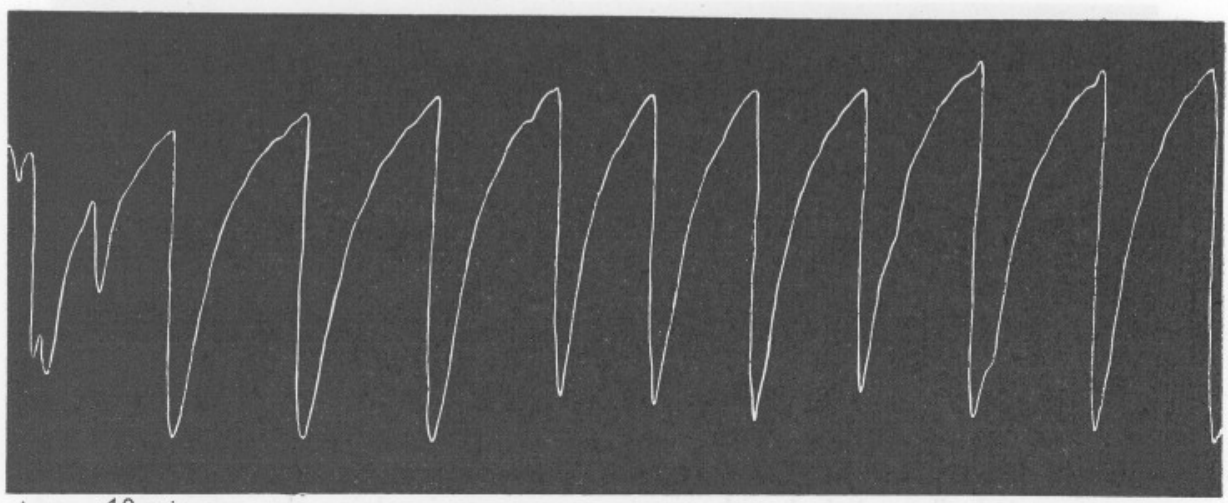

B

Fig. 4. Activity of simple ring preparations of Calliactis column. A, Preparation taken from submarginal region. $40 \mathrm{~min}$ record of activity without stimulation followed by period of $35 \mathrm{~min}$. during which electrical stimuli (Io stimuli at $\mathrm{I} \cdot \mathbf{2} \mathrm{sec}$ ) were applied at 12 and $26 \mathrm{~min}$ (arrows). The slow delayed contractions in response to stimuli are preceded by small contractions on the stimuli probably due to residual sphincter tissue in the preparation. B, Preparation taken from lower region of column. Record of activity during $2 \frac{1}{2} \mathrm{~h}$ without stimulation. 
therefore, on nervous, or perhaps intramuscular conduction. In the bigger movements involving all three loops, the submarginal region almost always took the lead. It began to move from 30 sec to I min before the sphincter and about I min or more before the loop from the lower part of the column. In the latter, independent movements frequently occurred. Fig. 5 shows records which illustrate these points. The general picture is similar to that found by Batham \& Pantin (1954) in Metridium, where there is a gradient of spontaneity from the sphincter to the pedal edge, though Calliactis is more
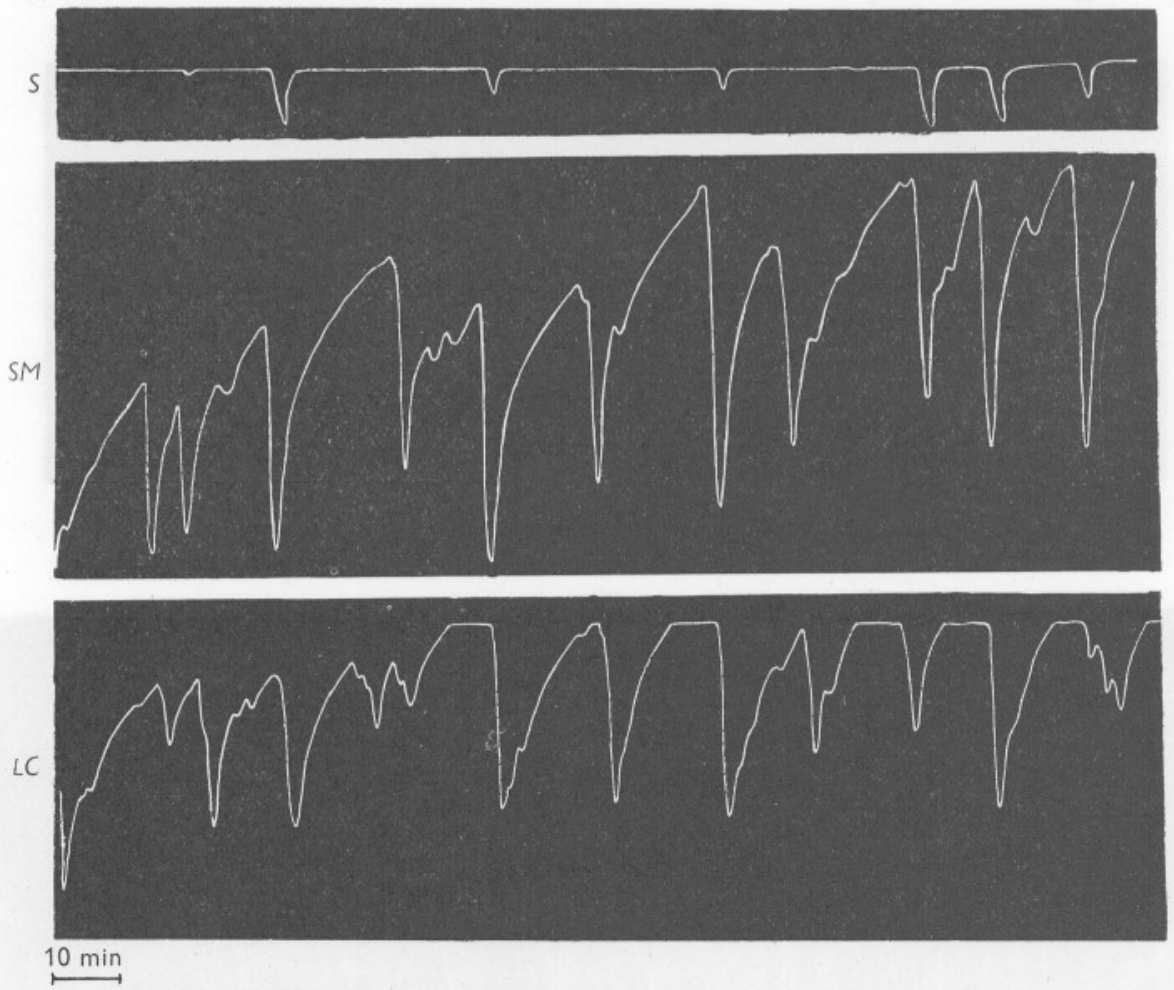

Fig. 5. Three-ring preparations of Calliactis with connecting strip of column recorded over $2 \frac{1}{2}$ h. $S$, sphincter ring attached to spring lever; $S M$, submarginal ring; and $L C$, lower column ring attached to weighted levers. The sphincter movements here recorded are slow contractions and all are linked with certain movements involving the other rings and beginning with the submarginal ring.

consistent in showing the submarginal region leading the co-ordinated movements. From the kymograph records of preparations alone, one might postulate that the pace-maker for these movements is located in the submarginal region, but we must recall that in the whole animal, a marginal twitch, often too small to be recorded, frequently precedes the main recorded 
movement. Therefore, the location of the pacemaker must remain an open question.

The effects of stimuli on the triple-loop preparations also showed the submarginal region taking the lead in the slow responses to stimuli at frequencies of $6 . \mathrm{I}$ and $7.5 \mathrm{sec}$. Even when the electrodes were nearer to the loop at the cinclide level, the submarginal loop began to contract about $30 \mathrm{sec}$ before either of the other two loops.

\section{Special experiment on five Calliactis}

The results presented so far refer to an assortment of Calliactis studied at different times and places. In order to obtain more definite information based on a few animals studied in detail, an additional experiment was set up. Five Calliactis on plates were placed in a bath and visual, photographic (still) and film records of their activities were made over a period of 3-4 weeks. They were fed once weekly with chopped Mytilus muscle and the sea water was changed one day after feeding. After thus observing the animals under aquarium conditions, each one was removed in turn and by means of threads inserted into the marginal, submarginal and cinclide regions of the column, 3-lever kymograph records were taken to show the movements of the whole animals over periods of about 2 days. After this, 3-loop preparations were made from each animal (after $4-6 \mathrm{~h}$ anaesthesia in $0.4 \mathrm{M}-\mathrm{MgCl}_{2}$ ) and their movements recorded on the same levers as long as the preparations survived in good condition, usually $\mathrm{I}_{2}-2$ days.

One feature of the observations was that each animal was found to have certain individual characteristics of attitude, posture and activity which it displayed throughout the experiment. The animals are shown in Fig. 6, and they were labelled A to E from left to right. Animal A usually kept its column moderately extended and its disk depressed below the level of the margin. Its tentacles were well extended but rather limp and trailing, and from the amount of movement of the margin, the number of closures observed, and the frequency of peristalsis, it was designated 'active'. In animal B the tentacles invariably rose stiffly from the margin, the column was always elongate and erect, with the margin kept in a near-vertical position. In this animal, activity was intense for short periods, with long periods of apparent immobility in between, and it was therefore described as 'intermittently active'. Calliactis C showed an asymmetrical posture, with the tentacles and margin slightly lifted on one side, Calliactis $\mathrm{D}$ a squat posture, with a deeply depressed disk and very short column, and Calliactis $\mathrm{E}$ an erect posture with almost no basal expansion, and a disk tending to project above the margin. Calliactis $\mathrm{C}$ was very active from time to time and so was classed as 'intermittently active'; Calliactis $\mathrm{D}$ and $\mathrm{E}$ moved infrequently and showed little variation in shape and were described as 'inactive'.

Photographs $a-f$ in Fig. 6, taken at I min intervals, illustrate the characteristic 


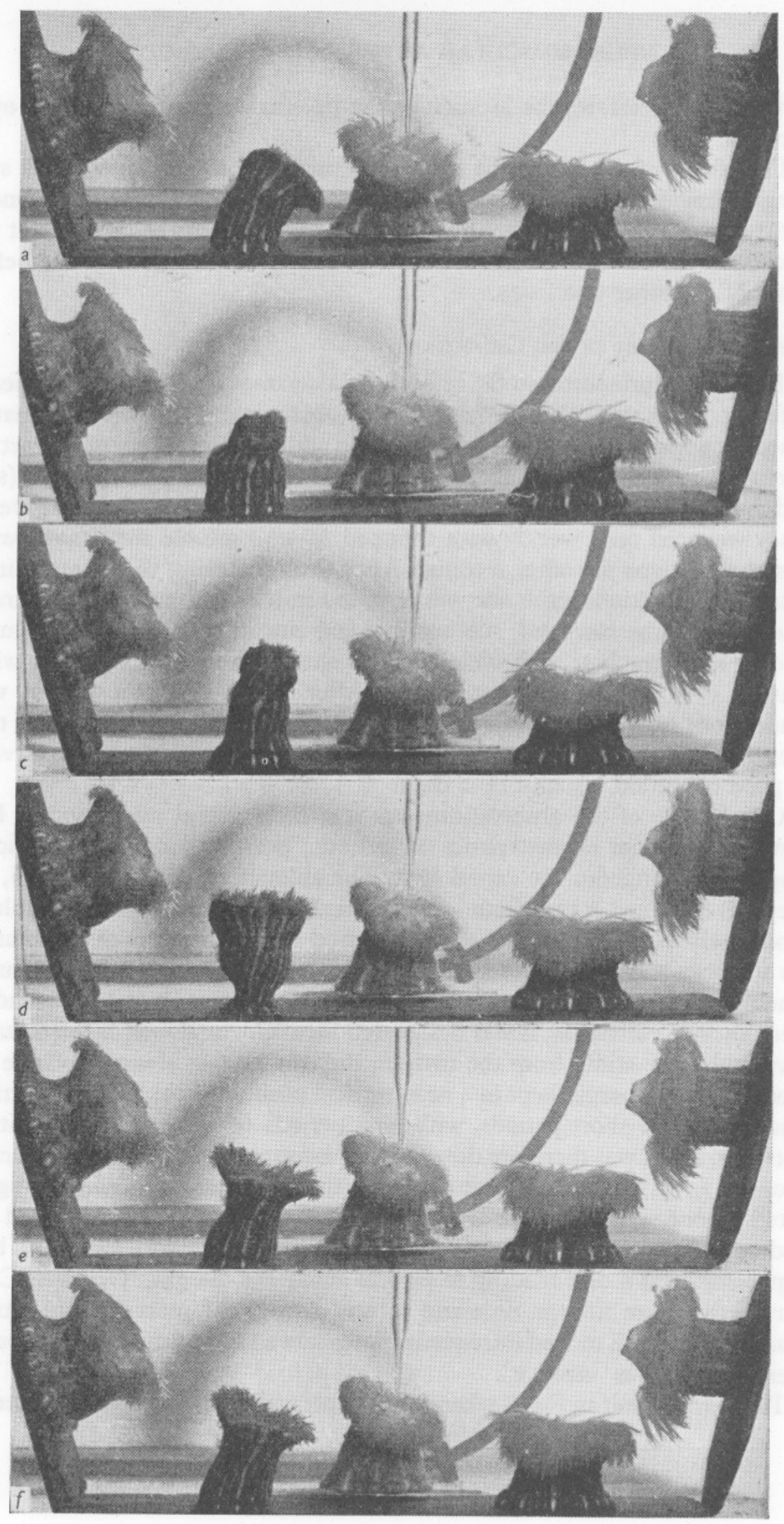

Fig. 6. For legend see opposite page. 


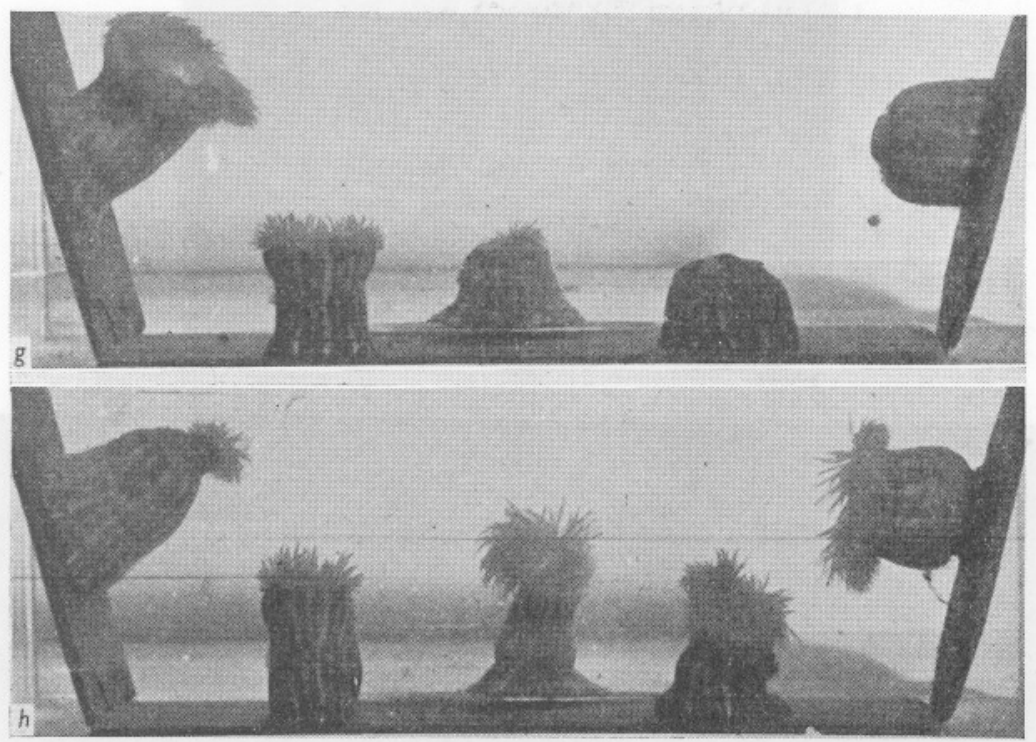

Fig. 6. Group of five Calliactis used in experiments described on pp. 797-803. Animals designated A, B, C, D, E from left to right. $a-f$, photos taken at intervals of I min showing 'normal posture' of animals A, C, D and E and active phase in animals B. $g$, 'normal posture' of animal B and active phases in animals A, C, D and E. $h, 2 \frac{1}{2}$ min after feeding.

postures of Calliactis A, C, D, E, and an activity cycle in Calliactis B corresponding to the description given earlier. The position in (b) was produced by a slow contraction of the margin which was maintained while the column was extended $(c)$. Positions $(d)$ and $(e)$ marked the beginning and end of a peristaltic wave in a subsidiary cycle. The characteristic posture of Calliactis B is shown in $(\mathrm{g})$. Under normal conditions, not more than I or 2 of these animals were active at once. Photograph $(g)$, in Fig. 6 shows one instance, however, when all these animals except Calliactis B were either closed or in active movement, in two cases due to defaecation. Feeding, as might be expected, initiates an outburst of activity which goes on for at least $15 \mathrm{~min}$. Several closing movements take place in quick succession and various distorted or asymmetrical positions are taken up before the animal returns to its normal posture and behaviour pattern. Photograph $(h)$ shows the group $2 \frac{1}{2}$ min after feeding. As far as could be seen, the normal rhythmic cycles were resumed within an hour or two, and were continued without marked change until defaecation initiated another outburst of activity about 2 days later.

Three-lever kymograph records of the activities of three of these Calliactis (A, C and E) and of 3-loop preparations made from them are shown in Fig. 7. These show the normal patterns of activity in periods outside feeding cycles lasting for 2 or $3 \mathrm{~h}$. The descriptions 'active', 'intermittently active' and 
A

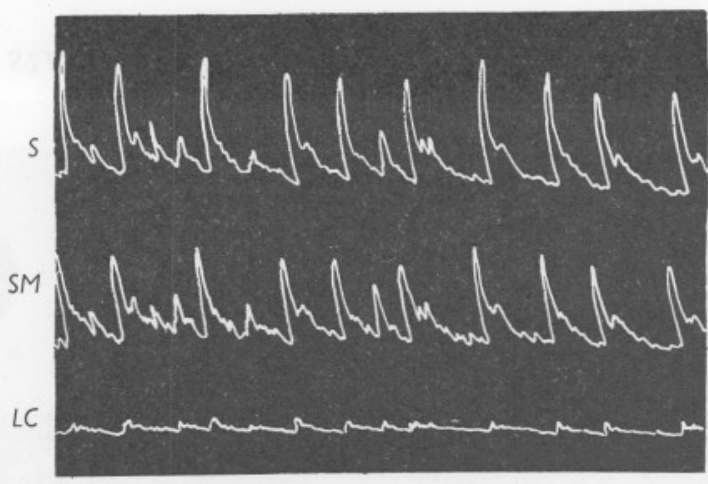

$-1$

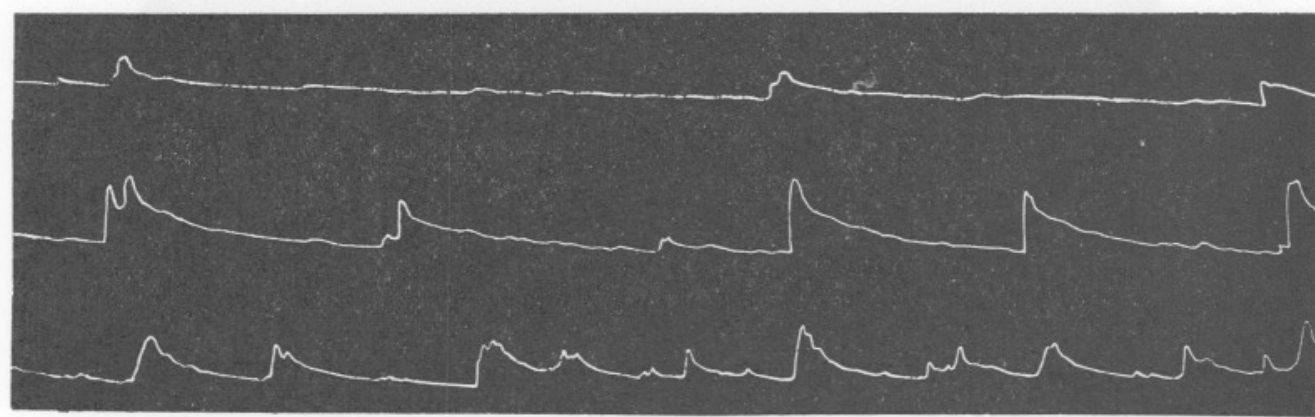

$\longmapsto$

B
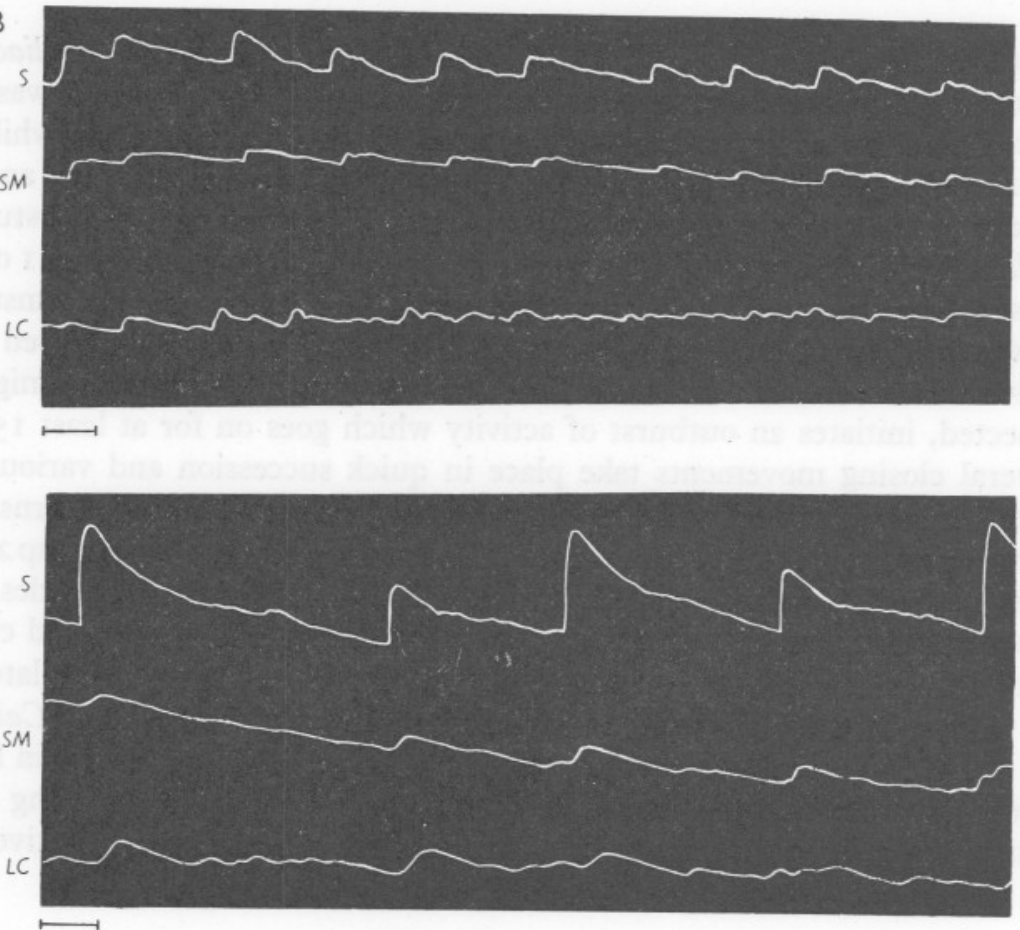

Fig. 7. For legend see opposite page. 

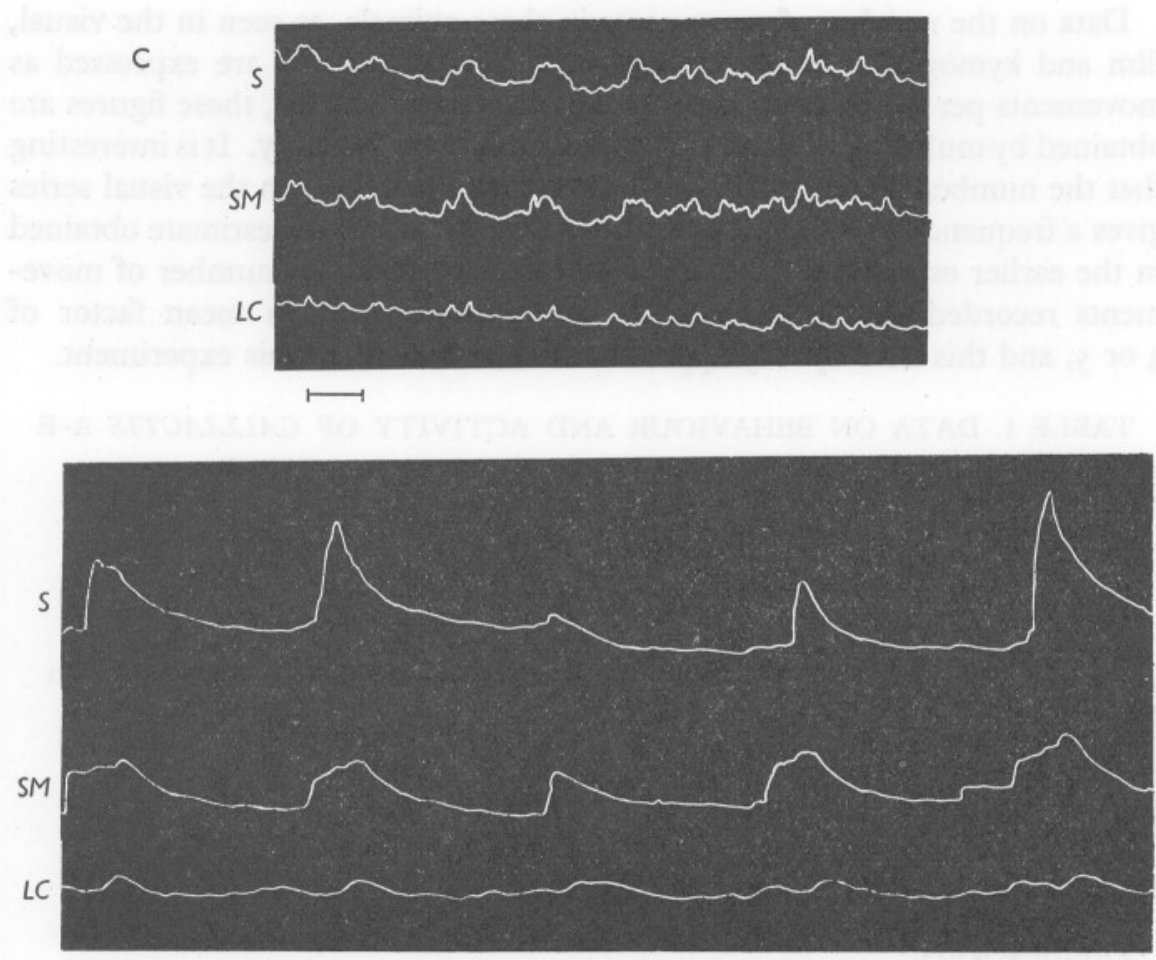

Fig. 7. Records of activity of intact Calliactis from the group of five experimental animals (cf. Fig. 6), with threads attached to marginal, submarginal and lower column regions, and records of three-loop preparations made later from the same animals. A, Intact Calliactis A (cf. Fig. 6) for $2 \mathrm{~h}$ and its 3-loop preparation for $4 \mathrm{~h}$ (7 days later); B, intact Calliactis C for $3 \mathrm{~h}$ and its preparation for $3 \mathrm{~h}$ ( 4 days later); $\mathrm{c}$, intact Calliactis $\mathrm{E}$ for $2 \mathrm{~h}$ and its preparation for $4 \mathrm{~h}$ (6 days later). S, SM and $L C$ indicate tracings from sphincter, submarginal and lower column regions in each recording. The scales represent a period of $10 \mathrm{~min}$.

'inactive', which were applied to Calliactis A, C and E respectively from their behaviour over many days, are seen here to apply also to some extent to their tracings.

It is not easy to distinguish between active and passive movements at a given region in the intact animal. Big movements of the animal at any level must lead to passive movements of a lever attached to a neighbouring level, and thus the marginal and submarginal levers usually show more or less identical movements in the upper tracings in Fig. 7. In four of the five animals A-E, the submarginal region took the lead in all movements except those which began with a quick contraction, but in animal C, the regular movements began with the margin. Near the base there were many independent movements though the amplitude of these was generally much smaller than at the other levels. 
Data on the number of movements in these animals, as seen in the visual, film and kymograph records, are given in Table I. They are expressed as movements per $24 \mathrm{~h}$, though for the film and visual records, these figures are obtained by multiplying up results for 6 and $20 \mathrm{hr}$ respectively. It is interesting that the number of marginal closing movements observed in the visual series gives a frequency of about $\mathrm{I}$ per $\mathrm{h}$ which is very close to the estimate obtained in the earlier experiment. But this figure falls short of the number of movements recorded in the film and on the kymograph by a mean factor of 4 or 5, and this descrepancy applies to all the animals in this experiment.

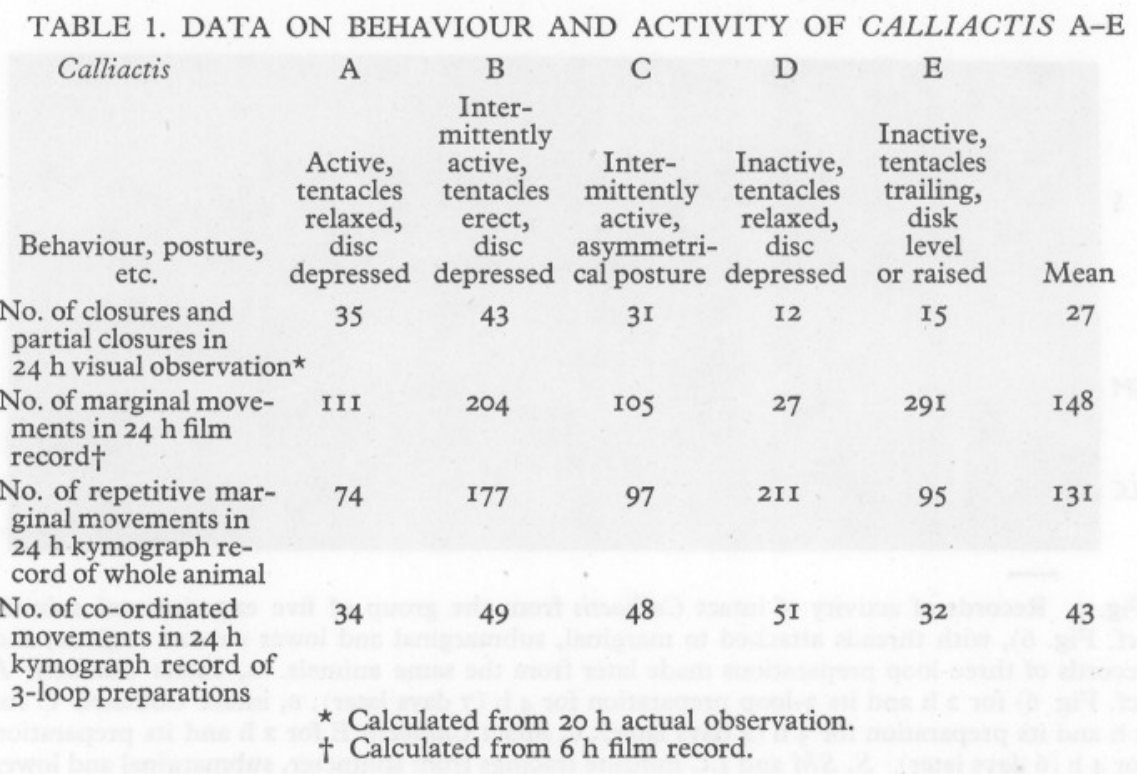

Difficulties in relating kymograph records to visually observed activity were encountered earlier, and it was hoped that the experiment with five Calliactis would clarify the matter. Instead, it has only emphasized the individual and phasic differences in the form and frequency of repetitive activity. In Calliactis C, D and E, the basic pattern of activity consists of movements hardly visible to the eye, appearing as slight twitches in the film and as small movements of the kymograph levers (Fig. 7 B, C). In Calliactis A and B, movements like those in the rhythmic cycles described above, which are associated with fairly conspicuous changes of shape or partial closure, dominate the kymograph records (Fig. $7 \mathrm{~A}$ ), but with a frequency two or three times as great as the same movements in the visual observations. It is unlikely that such movements would have been overlooked, so it must be assumed that the kymograph records in A and B coincided in time with phases of exceptional activity. 
The functions of these movements can only be surmised. However, from the changes of shape and volume accompanying the more conspicuous activity cycles, it might be inferred that some water is expelled from the coelenteron and replenished by fresh water at such times. The smaller repetitive movements of the levers seen in Fig. 7 B and $\mathrm{C}$ are due to small movements in the animal which lift the margin and constrict the submarginal region and other parts of the column. These movements are probably carrying out minor pumping and circulating activities, and the pattern is interrupted only at long intervals by the larger movements and closures which could provide for an exchange of water on a bigger scale.

The records of preparations made from these animals differ from those of the whole animals in many respects (Fig. 7). The frequency of the coordinated movements is much reduced and, although much closer to the frequency of closing movements as observed visually (Table I), the preparations show much more regularity. Four out of the five preparations examined gave movements that were very much larger than the movements at the same level in the intact animal, though as the figure shows, the enhancement was not always the same at all levels. However, this may only mean that as preparations the loops were freer to move than in the intact column.

It is interesting that in the preparations, the individual differences noted in the intact animals tend to disappear. The timing and general form of activity in these five preparations were not very dissimilar from one animal to another and bore little relation to the record taken from the whole animal. In the co-ordinated and independent activities of the different loops, these preparations behaved like the preparations described earlier, the submarginal loops being the first to move in all except Calliactis $\mathrm{C}$, and the loops at the level of the cinclides showing many movements of their own.

\section{DISCUSSION AND CONCLUSIONS}

The activities observed in Calliactis provide another example of an apparently almost immobile animal showing a great deal of co-ordinated behaviour occurring at a very slow time scale. But there is nothing simple or stereotyped about these activities. Patterns of behaviour in Calliactis are extremely variable with individual differences amongst animals and phasic differences in single animals at different times.

All the observations indicate that in the aquarium the quick closing response is a relatively infrequent occurrence, but it may happen more frequently when the animal is living on the shell with Eupagurus. Inconspicuous slow movements comprise most of the neuromuscular activity of the animal, and even a good deal of the activity of marginal sphincter itself. In the study of actinian nerve and muscle, work on the physiology of the slow activities is 
obviously very important. Quick facilitated contractions, notwithstanding their many interesting physiological features, are highly specialized activities in which some of the more typical and general properties of the actinian neuromuscular system may be obscured.

We feel that our observations should be regarded chiefly as another contribution to the growing literature on slow-periodic activities in invertebrate neuromuscular systems. Although the evidence from preparations suggests that these activities are co-ordinated, it is difficult to envisage appropriate mechanisms of conduction in the nerve net or intramuscular slow conduction processes to account for the exceptionally slow passage of the co-ordinated movement through the animal. But slow periodicity is a feature of many processes in which neuromuscular activity is not generally regarded as being involved. Dr L. J. Hale of Edinburgh has made a film of the growth of an Obelia colony by time-lapse cinematography, and the results seem remarkable to us and highly relevant to the phenomena seen in the activities of anemones. Apparently the growth of the colony is a pulsating process in which advancing movements are followed by partial withdrawals in a rhythmical way. Moreover, the timing of these 'beats' or 'pushes' is of the same order as the timing of the recorded 'beats' in both Metridium and Calliactis, occurring every few minutes (Hale, personal communication). Perhaps the notion that there is an underlying slow periodicity in the lives of certain organisms, involving their growth and development as well as their behaviour, is one that might be borne in mind in this connexion.

Slow periodic activities are now known to be involved in the behaviour of widely different groups of animals, in burrowing worms (Wells, 1950), in tunicates (Hoyle, I952), as well as sessile coelenterates. Although these activities are generally described as spontaneous because they often have no obvious external or internal causes, and particularly because they are often seen in isolated preparations, they have many features that deserve further investigation. Koshtoyantz \& Smirnova (I955) reported a relationship between the availability of sulphydryl groups and the initiation and maintenance of slow rhythmic movements in Actinia. We repeated their experiments on ring preparations of Calliactis but did not find that cadmium chloride inhibited, or that cysteine re-started, slow periodic movements in the way they had described. Yet this approach could be very useful if applied generally to this problem. Experiments aiming to reveal the properties of these spontaneous slow periodic activities by testing the action of a wide range of treatments, using metabolic as well as neuromuscular agents, might produce results throwing a great deal of light on this still obscure corner of comparative physiology.

We acknowledge with very warm thanks the help we received from the Director and Staff of the Plymouth Laboratory where much of the work was 
carried out. We have also had invaluable assistance from $\mathrm{Mr}$ W. Brackenbury, who did the photography, and from Mr Norman MacQueen of the MacQueen Film Organization, who took the film sequence. One of us (M.N.) also thanks Prof. P. B. Medawar, F.R.S., for the facilities and hospitality received at the Zoology Department, University College, London, during several months spent there in $1954-55$ as a visiting research worker from Canada.

\section{SUMMARY}

Features in the behaviour of the sea anemone, Calliactis parasitica, as seen in visual, photographic and kymograph records, are described.

As well as occasional and aperiodic closures, sometimes maintained for several hours, this anemone shows slow, complex, co-ordinated activity cycles whose main components are repeated at fairly regular intervals. These occur several times per hour in most animals, but their form and frequency show many individual and phasic variations. Slow movements of the marginal sphincter contribute to these activities.

Contractions of the submarginal region generally lead the slow co-ordinated movements in whole animals and 3-loop preparations, but tiny marginal twitches often precede them in whole animals. Loops and ring preparations taken near the base show most independent activity.

\section{REFERENCES}

Batham, E. J. \& Pantin, C. F. A., I950a. Muscular and hydrostatic action in the sea-anemone Metridium senile (L.). F. exp. Biol., Vol. 27, pp. 264-89.

- 1950 b. Inherent activity in the sea-anemone Metridium senile (L.). F. exp. Biol., Vol. 27, pp. 290-301.

- 1954. Slow contraction and its relation to spontaneous activity in the seaanemone Metridium senile (L.). F. exp. Biol., Vol. 31, pp. 84-103.

Chapman, G., 1949. The mechanism of opening and closing of Calliactis parasitica. F. mar. biol. Ass. U.K., Vol. 28, pp. 64I-50.

Hoyle, G., 1952. The response mechanism in ascidians. F. mar. biol. Ass. U.K., Vol. 3I, pp. 287-306.

Koshtoyantz, Ch.S. \& SMirnova, N. A., I955. On the nature of periodic activities in sea anemones. Dokl. obsch. Sobr. AK. Nauk S.S.S.R., Vol. 104, pp. 662-5. (In Russian.)

Pantin, C. F. A., I935 a. The nerve net of the Actinozoa. I. Facilitation. F. exp. Biol., Vol. I2, pp. II $19-38$.

- $1935 b$. The nerve net of the Actinozoa. II. Plan of the nerve net. F. exp. Biol., Vol. 12, pp. 139-55.

- 1940. Demonstrations of behaviour in the lower animals. Sch. Sci. Rev., Vol. 84, p. Ir 32 .

Ross, D. M., 1957. Quick and slow contractions in the isolated sphincter of the seaanemone, Calliactis parasitica. F. exp. Biol., Vol. 34, pp. II-28.

Wells, G. P., I950. Spontaneous activity cycles in polychaete worms. Symp. Soc. exp. Biol., Vol. 4, pp. 127-42. 